\title{
Numerical investigation of volute tongue design on hemodynamic characteristics and hemolysis of the centrifugal blood pump
}

\author{
Ibrahim Basar Aka ${ }^{1}$ (1) $\cdot$ Caglar Ozturk $^{2}$ Ismail Lazoglu²
}

Received: 2 May 2020 / Accepted: 4 January 2021 / Published online: 9 January 2021

(C) The Author(s) 2021 OPEN

\begin{abstract}
In the design of rotary blood pumps, the optimization of design parameters plays an essential role in enhancing the hydrodynamic performance and hemocompatibility. This study investigates the influence of the volute tongue angle as a volute geometric parameter on the hemodynamic characteristics of a blood pump. A numerical investigation on five different versions of volute designs is carried out by utilizing a computational fluid dynamics (CFD) software ANSYSFLUENT. The effect of volute tongue angle is evaluated regarding the hydrodynamic performance, circumferential pressure distribution, the radial force, and the blood damage potential. A series of volute configurations are constructed with a fixed radial gap (5\%), but varying tongue angles ranging from 10 to $50^{\circ}$. The relative hemolysis is assessed with the Eulerian based empirical power-law blood damage model. The pressure-flow rate characteristics of the volute designs at a range of rotational speeds are obtained from the experimental measurements by using the blood analog fluid. The results indicate an inverse relationship between hydraulic performance and the tongue angle; at higher tongue angles, a decrease in performance was observed. However, a higher tongue angle improves the net radial force acting on the impeller. The pump achieves the optimized performance at $20^{\circ}$ of the tongue angle with the relatively high hydrodynamic performance and minor blood damage risk.
\end{abstract}

Keywords Centrifugal blood pump · Hemolysis · Numerical simulation · Volute design $\cdot$ Volute tongue

\section{List of symbols}

$D \quad$ Blood damage index

$T \quad$ Scalar Shear stress $(\mathrm{Pa})$

$\mathrm{Hb}$ Hemoglobin content $(\mathrm{g} / \mathrm{L})$

$d H b \quad$ Damaged hemoglobin content $(\mathrm{g} / \mathrm{L})$

$\delta \quad$ Radial gap (\%)

$\mathrm{N}_{\mathrm{s}} \quad$ Pump specific speed $(\mathrm{m} 3 / \mathrm{h}, \mathrm{m})$

$n \quad$ Rotational speed (rpm)

$h \quad$ Pressure head (m)

$Q \quad$ Flow rate $(\mathrm{L} / \mathrm{min})$

\section{Introduction}

Continuous-flow cardiac assist pumps have been successfully used to treat end-stage heart failure patients as destination therapy, bridge-to-transplantation or bridgeto-recovery [1-3]. However, hemolysis reduction is still a significant design challenge for blood pumps [2, 3]. Even though the impeller geometry plays an essential role in the overall performance of a centrifugal blood pump [4], the volute structure is equally critical for assuring steady flow lines and good kinetic energy to potential energy conversion for achieving greater hydraulic performance and a lower hemolysis risk [5, 6]. Moreover, the uniformity of the pressure distribution around the impeller is a crucial parameter for designing non-contact bearings for

Ibrahim Basar Aka, basar.aka@bilgi.edu.tr | ${ }^{1}$ Mechatronics Engineering Department, Istanbul Bilgi University, Istanbul 34060, Turkey. ${ }^{2}$ Manufacturing and Automation Research Center, Koç University, Istanbul 34450, Turkey. 
centrifugal pumps. Non-axisymmetric nature of the volute geometry generates a non-uniform pressure distribution around the impeller, thus inducing a radial force critical for the magnetic suspension of pumps. Several researchers have studied the effect of volute geometry on the performance of centrifugal pumps at larger scales to address this problem $[7,8]$. The volute tongue region has been particularly studied in various engineering fields for improving the pump endurance, bearing life, noise reduction, magnetic levitation and the flow separation [7]. However, recent studies on rotary blood pumps have mostly focused on the impeller geometry and blade parameters to optimize the pump performance and minimize the blood damage risk $[4,9]$. To date, a few researchers have numerically investigated the hydraulic effect of volute parameters such as the volute tongue in rotary blood pumps [5], yet in these studies the effect of volute tongue position on the hemolytic characteristics is missing.

Computational fluid dynamics (CFD) has been broadly used for the design and optimization of rotary blood pumps [9-11]. This method is a reliable technique to investigate the overall flow characteristics, and to simplify the development period of the device, reducing the time and cost of experimentation [2,3]. As the blood damage potential has recently been incorporated into these CFD analyses, various numerical models have been adopted by several research groups to estimate the hemolysis and optimize the design of rotary blood pumps $[10,11]$. Although the researchers are still questioning the accuracy of the computational blood damage models for predicting the absolute level of hemolysis, it has become an accepted method for calculating the relative hemolysis level for the design optimization [10, 12]. The ISO 14,708-5 standard also suggests using the CFD simulations for evaluating the relative design changes rather than determining the absolute quantities [13].

In this study, five volute configurations were constructed in a virtual environment with the tongue angles (TA) ranging from $10^{\circ}$ to $50^{\circ}$ in $10^{\circ}$ increments and numerical simulations were conducted to evaluate the effect of tongue position. Firstly, the hydrodynamic performance and the relative hemolysis characteristic were numerically investigated by employing the Eulerian blood damage estimation approach in a CFD model. In the next step, the presented models were rapid-prototyped and a hydraulic experiment was conducted with a blood analog fluid to analyze their pressure-volumetric flow rate characteristics. Finally, the hemolytic and hydraulic performance of volute geometries were compared based on the in-silico findings and in-vitro measurements. The objective of this study is to investigate the influence of the tongue angle on the pump hemodynamics and optimizing the tongue design for a better matching volute and impeller region.

\section{Materials and methods}

\subsection{Pump geometry}

The physical design of the pump is comprised of a rotating impeller with five blade-splitters, an inlet guided by three straight blades, a volute converting the kinetic energy to the potential energy and two ceramic bearings for contact regions. The complete pump assembly is shown in Fig. 1. The pump employs the concept of magnetic coupling with the magnets embedded inside the impeller. A miniature DC-motor drives the impeller through magnetic coupling. The pump used in this study is $25 \mathrm{~mm}$ in height, $51 \mathrm{~mm}$ in length and houses a $45 \mathrm{~mm}$ diameter impeller inside. The major specifications of the pump are summarized in Table 1. The pump is designed to operate at a rotational speed of $2000 \mathrm{rpm}$, targeting a $5 \mathrm{~L} / \mathrm{min}$ pump flow rate and $100 \mathrm{mmHg}$ head pressure according to the clinical use at the hospitals[14, 15]

\subsection{Volute design}

The flow pattern inside a centrifugal pump is quite intricate, and the pump characteristics are critically affected by the geometrical constraints such as impeller-volute interactions [7]. The pump geometry was designed using a CAD software (Siemens NX 10.0, Siemens AG, Munich, Germany) with a configuration to create an adjustable impeller-volute region. For the present study, the constant mean velocity method was used to design the volute geometry according to the Stepanoff design rule [16]. The pump specific speed (Ns) was set to $790\left(\mathrm{~m}^{3} / \mathrm{h}, \mathrm{m}\right)$ for all models based on Eq. (1). The tongue shape and the circumferential angular position (tongue angle, $\theta$ ) were shown in Fig. 2 .

$N_{s}=\frac{n \sqrt{Q}}{h^{3 / 4}}$

Radial gap $(\delta)$ between the impeller periphery and volute tongue was identified as a percentage of the impeller outer diameter based on the guideline [16].

$\delta=\frac{D 3-D 2}{D 2} \times 100 \%$

A total of five volute models, ranging from $10^{\circ}$ to $50^{\circ}$ tongue angles, were constructed for the specified pump geometry. The tip of the volute tongue was round, and the lowest volute tongue angle was defined at $10^{\circ}$. Angles less than $10^{\circ}$ were disregarded to avoid an excessive sharp corner on the tongue tip. As the tongue angle ranged from $10^{\circ}$ to $50^{\circ}$, the tongue thickness was varied based 

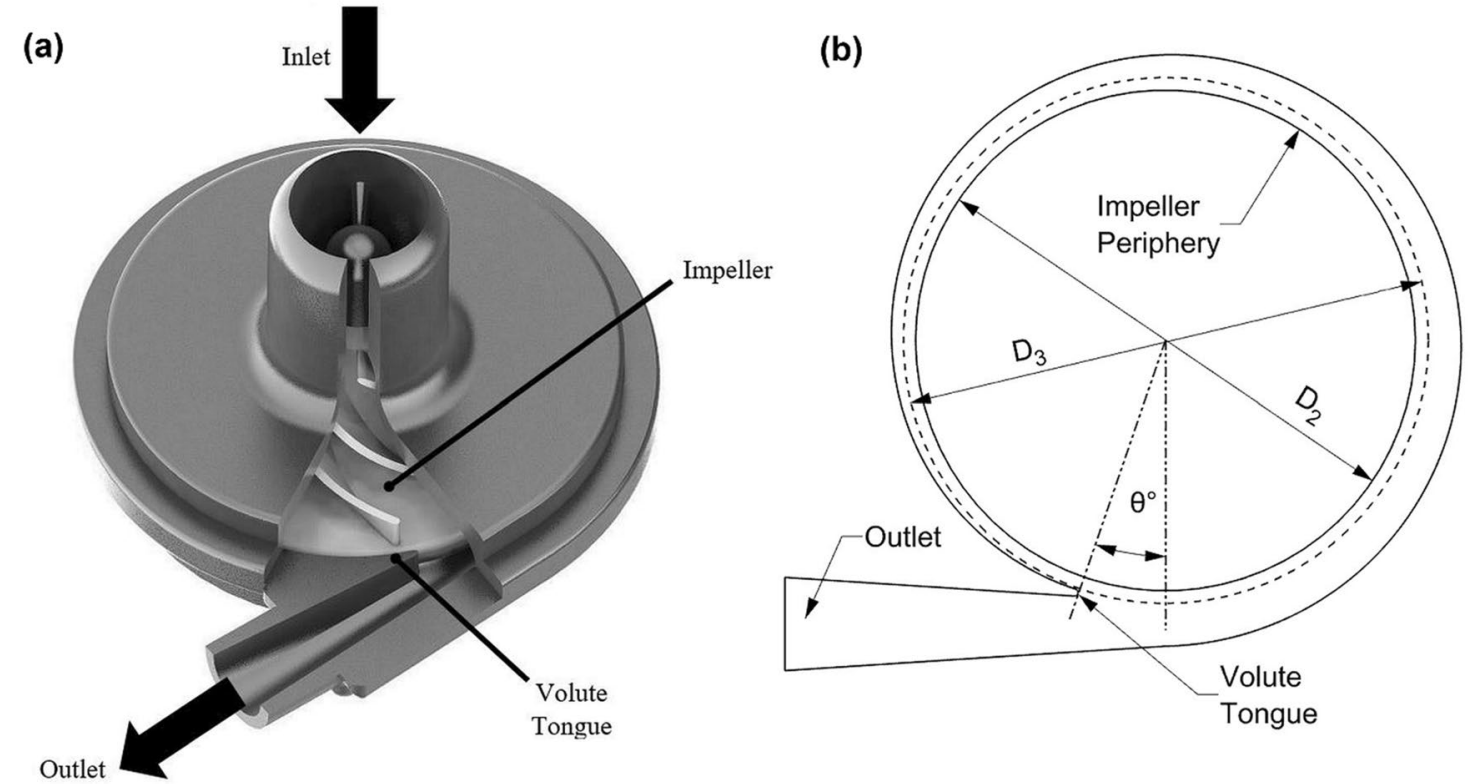

Fig. 1 a Centrifugal blood pump geometry. b Definition of the volute tongue position

Table 1 Main specifications of the pump

\begin{tabular}{ll}
\hline Parameter & Value \\
\hline Operating speed $(n)$ & $2000 \mathrm{rpm}$ \\
Specific speed $\left(N_{s}\right)$ & $790 \mathrm{~m}^{3} / \mathrm{h}, \mathrm{m}$ \\
Impeller inlet diameter $\left(D_{1}\right)$ & $12 \mathrm{~mm}$ \\
Impeller outlet diameter $\left(D_{2}\right)$ & $45 \mathrm{~mm}$ \\
Basic circle diameter $\left(D_{3}\right)$ & $47.3 \mathrm{~mm}$ \\
Volute inlet width & $6 \mathrm{~mm}$ \\
Number of blades & 5 \\
Number of blade splitters & 5 \\
Blade inlet angle & $36.5^{\circ}$ \\
Blade outlet angle & $36.5^{\circ}$ \\
Blade wrap angle & $120^{\circ}$ \\
\hline
\end{tabular}

on the diameter of the arc. The radial gap $(\delta)$ between the proposed volute designs was kept constant at $5 \%$ of the impeller outer diameter by using Eq. (2). The volute outlet had a circular cross-section with a $9 \mathrm{~mm}$ end diameter. The tongue position was characterized by only changing the tongue angle, and the relative positions of other geometrical components such as volute throat and impeller-volute regions were fixed.

\subsection{CFD method and boundary conditions}

In this study, we employed a previously developed CFD Model and this model was validated against previously published experimental study of the FDA centrifugal pump [4]. A commercial software (ANSYS Fluent 18.0,
ANSYS Inc., Pittsburgh, PA, USA) was used to model the flow pattern inside the blood pump. The sliding mesh method was applied between the rotating impeller and the stator casing to simulate the flow dynamics. The blood was modeled as an incompressible Newtonian fluid with $1050 \mathrm{~kg} / \mathrm{m} 3$ density and 0.0035 Pas dynamic viscosity. Although the blood is known as non-Newtonian fluid, it only exhibits non-Newtonian properties at low shear rates $(<100 / s)$ [17]. Hence, the Newtonian fluid characteristic is adopted for the blood flow due to the high shear rates inside the rotary blood pumps [10]. During the grid generation process, orthogonal quality, aspect ratio, and the skewness were assured to be in the required range.

Turbulence was simulated with the $k-\omega$ model by considering the mean $\gamma+<3$ value with appropriately sized cells near domain walls. The final meshes contained approximately 10 million cells. The convergence precision was set to $10^{-4}$ for the scaled residuals. The inlet and outlet ports were extended to a specific length to prevent boundary condition effects on the convergence. The impeller was set to complete one rotation in 100 steps indicating $3.6^{\circ}$ rotation for each step, and the CFD time step was adjusted to $3.00 \times 10^{-4} \mathrm{~s}$ accordingly. The inlet boundary condition was set to flow rate of $5 \mathrm{~L} / \mathrm{min}$, and the outlet pressure was set to $0 \mathrm{~Pa}$ as nominal values at $2000 \mathrm{rpm}$. The absolute pressure difference was monitored between the inlet and outlet port. Hydraulic efficiency and the net radial force acting on the impeller were numerically calculated. The physical quantities such as shear stress, pressure and the torque values on the rotation axis were monitored to detect the flow conditions after 3 
Fig. 2 The experimental setup showing all the major components

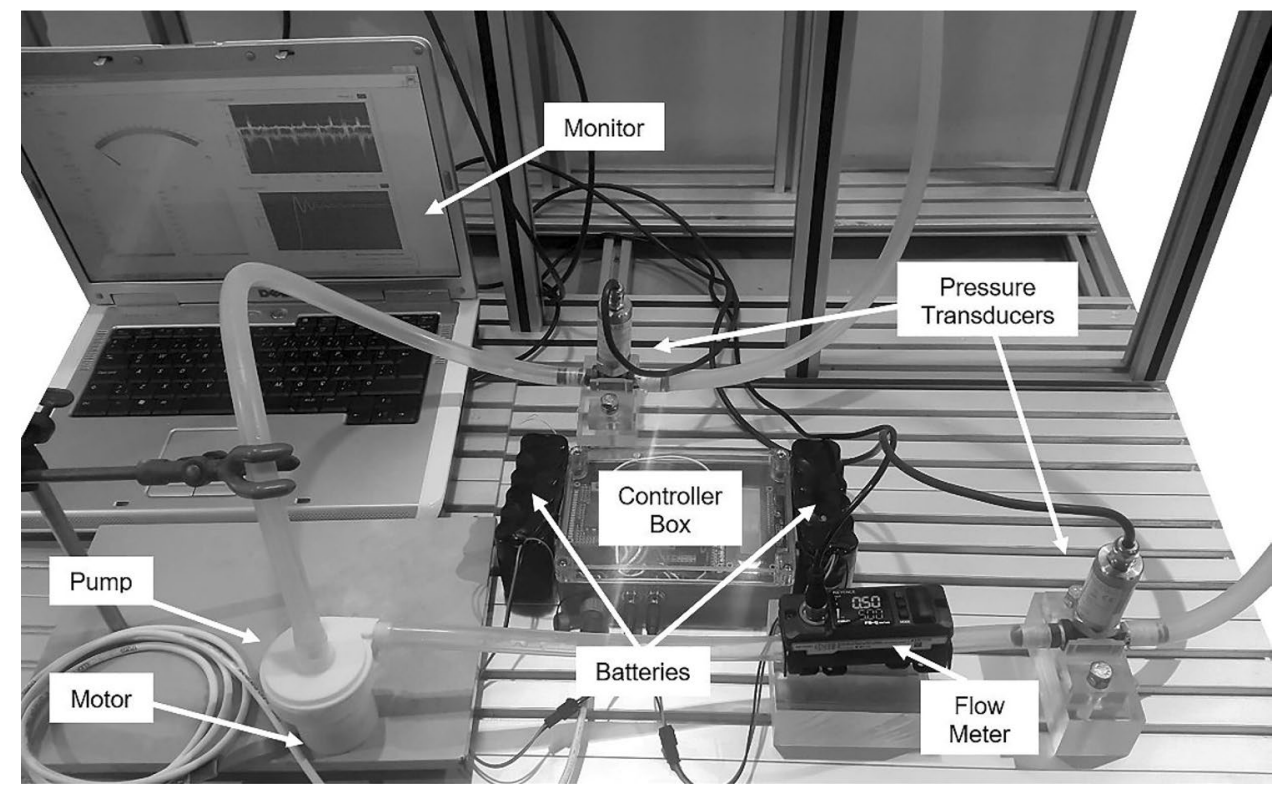

revolutions. User-Defined Functions (UDF) were compiled and used for the quantification of the blood damage.

\subsection{Hemolysis estimation}

An empirical power-law model has mostly been used to compute the hemolysis in blood pumps [10]. The hemolysis potential of the pump is quantified as a blood damage index (D) which indicates the increase in plasma-free hemoglobin. The power law model predicts the blood damage by correlating the plasma-free hemoglobin level with the shear stress and exposure time [18],

$D=\frac{\mathrm{dHb}}{\mathrm{Hb}}=C \tau^{\alpha} t^{\beta}$

where $C$, $a$ and $\beta$ are the constants obtained by postprocessing the experimental data, $\tau$ is the scalar stress, $t$ is the exposure time of blood cell, $\mathrm{dHb}$ represents the damaged hemoglobin content, and $\mathrm{Hb}$ indicates the hemoglobin content. As these constants vary based on the conducted experiment, several researchers have proposed different values [10]. The constants Heuser et al. $\left(C=1.8 \times 10^{-6}, \alpha=0.765, \beta=1.991\right)$ [19] suggest $(C=1.8 \times 10-6, a=0.765, \beta=1.991)$ were utilized in this numerical study due to the reported accuracy over experimental data [10].

$D=\frac{\mathrm{dHb}}{\mathrm{Hb}}=1.8 \times 10^{-6} \tau^{1.991} t^{0.765}$

Scalar shear stress (SSS) parameter can be found by the following expression [17]; $\tau=\left[\frac{1}{6} \sum\left(\tau_{i i}-\tau_{j j}\right)^{2}+\sum \tau_{i j}^{2}\right]^{\frac{1}{2}}$

The power-law equation was linearized to obtain the concentration of damaged blood $[20,21]$. This method directly computes the damage index from the velocity and the shear stress field by the volume integration over the entire flow domain. The time dependency of the power law was eliminated by the linearization $[20,21]$. The linearized blood damage index was then expressed as:

$\frac{\partial}{\partial t} \mathrm{D}_{l}=\left(\frac{\partial}{\partial \mathrm{t}}+\bar{u} . \nabla\right) D_{l}=\tau$

A hyperbolic equation was also obtained by taking the time derivative of Eq. (6) and applying the Eulerian transform $[20,21]$.

$$
D=\int_{\text {inlet }}^{\text {outlet }} 1.8 \times 10^{-6} \tau^{1.991} t^{0.765}
$$

\subsection{In vitro hydraulic test}

A hydraulic test loop was constructed to validate and compare the hydraulic performance of the models. The loop contains a pump prototype, two piezoelectric pressure transducers, an ultrasonic flowmeter, a DC motor, a motor controller box, a DAQ system, a total of $2 \mathrm{~m}$ length flexible polyvinyl chloride tubing (3/8-inch ID) and a reservoir. The pump prototypes were manufactured from $A B S$ plastic with a 3D printer (Ultra 3SP Envisiontec GMBH, Gladbeck, Germany). The brushless DC motor (Maxon 
Motor AG, Sachseln, Switzerland) was magnetically coupled to the impeller. A motor controller was implemented for controlling and reading the speed of the impeller. A water-glycerol mixture was prepared as a blood analog fluid (65\% water, $35 \%$ glycerol) at the room temperature $\left(25^{\circ} \mathrm{C}\right)$ in accordance with the CFD simulations. As the fluid traveled through the loop, a valve was used for adjusting the resistance to the flow. The flow rate was evaluated via the ultrasonic flowmeter (FD-Q 10C, Keyence Corp., Osaka, Japan) which was placed on the outlet tubing. Two piezoelectric pressure transducers (RAG25R0, Kistler Holding AG, Winterthur, Switzerland) were placed through the inlet and the outlet tubing. A DAQ card (LabView National Instruments, Austin, TX, USA) and the interface were used to collect pressure readings with a $10 \mathrm{kHz}$ sampling rate.

Table 2 Effect of the tongue angle on the hydraulic performance

\begin{tabular}{lccccr}
\hline Parameter & \multicolumn{7}{l}{ Tongue angle } & & & \\
\cline { 2 - 6 } & $10^{\circ}$ & $20^{\circ}$ & $30^{\circ}$ & $40^{\circ}$ & $50^{\circ}$ \\
\hline Pressure head (mmHg) & 102.2 & 99.1 & 95.3 & 96.8 & 93.2 \\
Hydraulic efficiency (\%) & 49.3 & 47.9 & 45 & 46.3 & 44.8 \\
Net radial force (N) & 0.33 & 0.3 & 0.29 & 0.22 & 0.2 \\
\hline
\end{tabular}

\section{Results}

The hydraulic and the hemolytic performances of five volute models were compared at the design point flow rate of $5 \mathrm{~L} / \mathrm{min}$. Table 2 illustrates the overall hydraulic performance of the models. The tongue angle was varied from $10^{\circ}$ to $50^{\circ}$ in $10^{\circ}$ increments. The pressure head and the hydraulic efficiency were observed to decrease correspondingly as the tongue angle increase. The poorest hydraulic performance was realized in the $50^{\circ}$ tongue angle with an efficiency of $44.8 \%$ and a pressure head of $93.2 \mathrm{mmHg}$, but the radial force was lowest at $0.2 \mathrm{~N}$ among all cases.

Pressure distributions of five different cases were compared in Fig. 3. The absolute pressure contours of the midvolute layer were plotted for all cases, respectively. A more uniform pressure field was observed around the impeller region at the $50^{\circ}$ tongue angle. Moreover, an increasing tongue angle reduced the net radial force acting on the impeller. The velocity vector fields were displayed in Fig. 4. The relative velocity field presents the flow interaction between the outlet and the impeller outflow. At a low tongue angle, the flow was observed as well-guided along the volute region through the outlet. However, the flow
Fig. 3 Absolute pressure contours in various tongue angles, a $10^{\circ}$, b $20^{\circ}$, c $30^{\circ}$, d $40^{\circ}$, e $50^{\circ}$

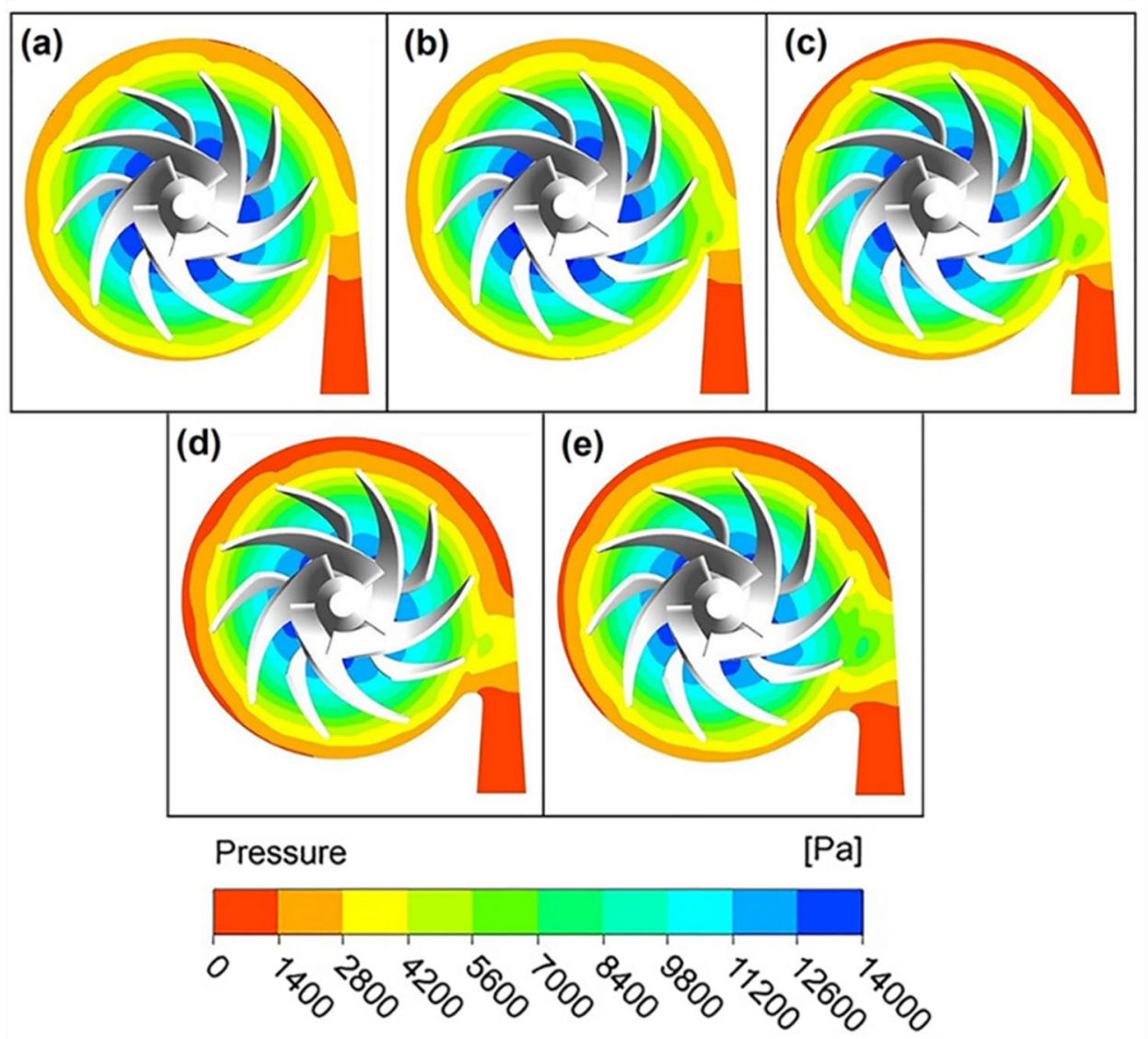

SN Applied Sciences A SPRINGER NATURE journa 
Fig. 4 Velocity vector field at different tongue angles. a $10^{\circ}$, b $20^{\circ}$, c $30^{\circ}$, d $40^{\circ}$, e $50^{\circ}$
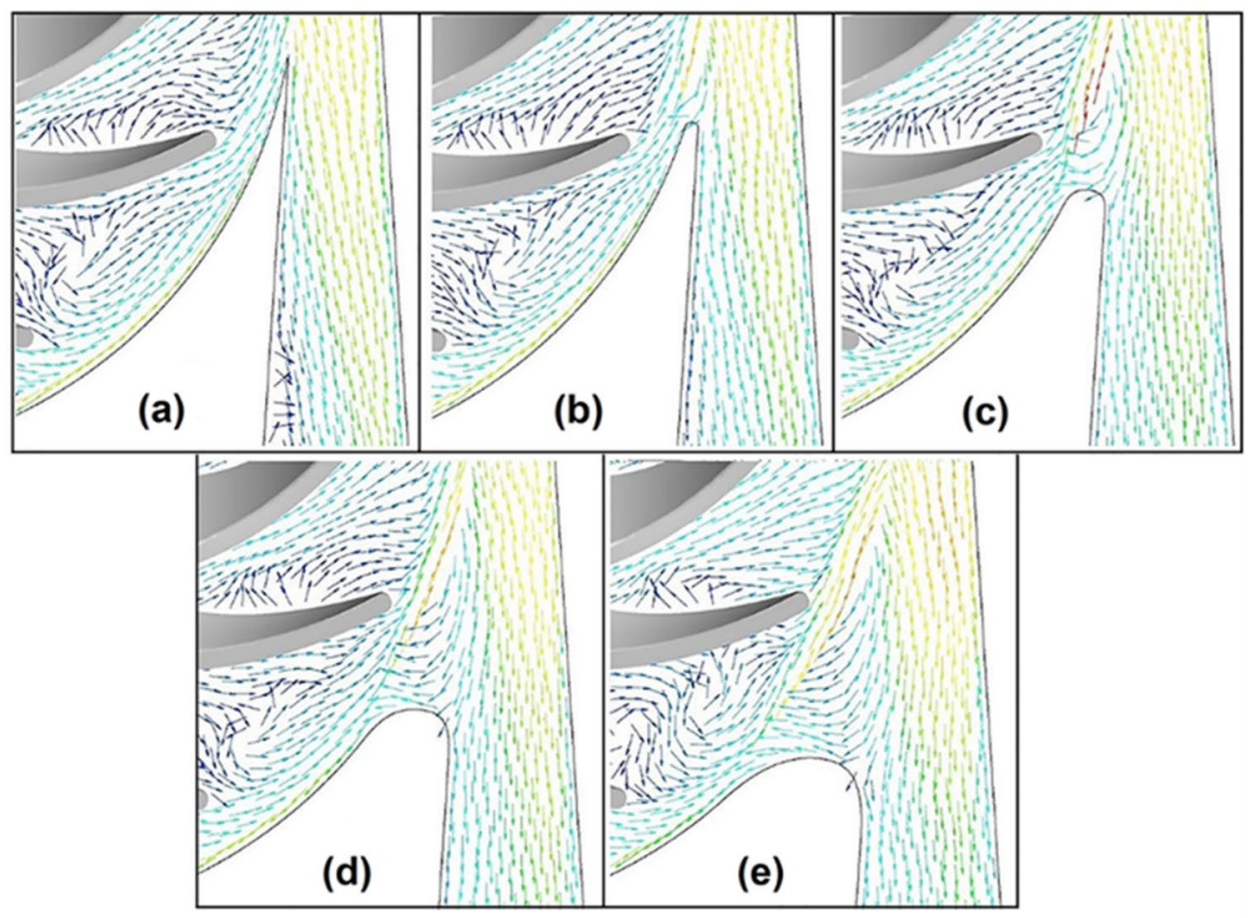

Velocity

$\left[\mathrm{m} \mathrm{s}^{\wedge}-1\right]$

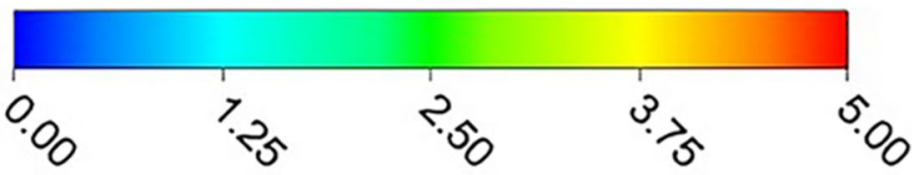

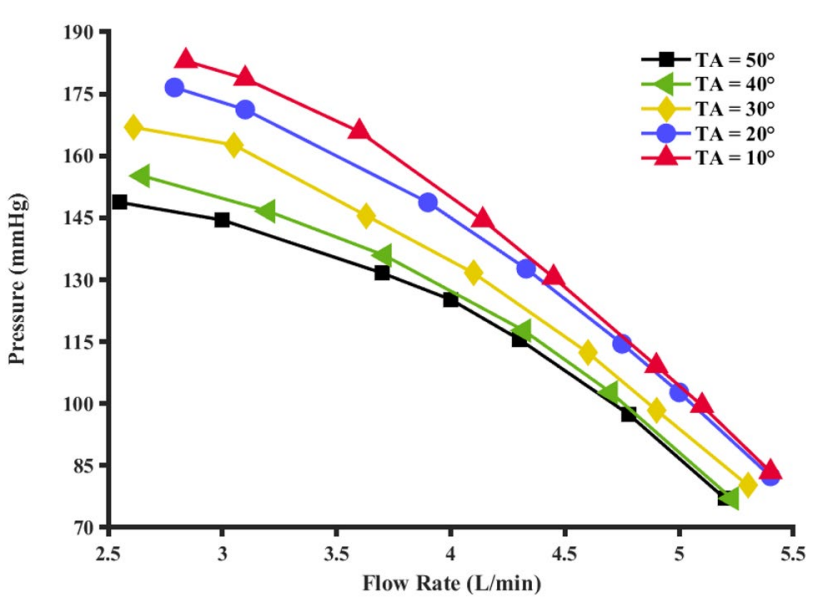

Fig. 5 Pressure-flow rate characteristics of five volute configurations at $2000 \mathrm{rpm}$. Volute tongue angles (TA) range from 10 to $50^{\circ}$

was separated through to the suction side of the blades as the tongue angle increased.

The findings of hydraulic experiments were also reported in Fig. 5. The pressure-flow performance was measured at $2000 \mathrm{rpm}$. The performance curves revealed that the configuration of $50^{\circ}$ tongue angle had the poorest hydraulic output, and the hydraulic performance was observed to be negatively influenced by a larger tongue angle. The SSS was also computed based on Eq. (5). High shear stress zones were mostly concentrated around the trailing edges of the blades and the volute tongue region. The maximum and averaged SSS in the entire flow domain were shown in Fig. 6.

The most extensive shear stress was observed at the $50^{\circ}$ of tongue angle, for which the maximum SSS was found to be $354.4 \mathrm{~Pa}$, and the average SSS was calculated as 13.9 Pa. By comparing the relative blood damage of various tongue angles in Fig. 7, the minimum Relative Hemolysis Index (RHI), indicated to be optimal for the $20^{\circ}$ tongue angle. The highest $\mathrm{RHI}$ was observed when the tongue angle was $50^{\circ}$ which means a greater possibility of blood damage for larger tongue angles.

\section{Discussion}

In general, the comparison of five models revealed that the major differences might be observed in the pump performance depending on the volute profile. Pressure 


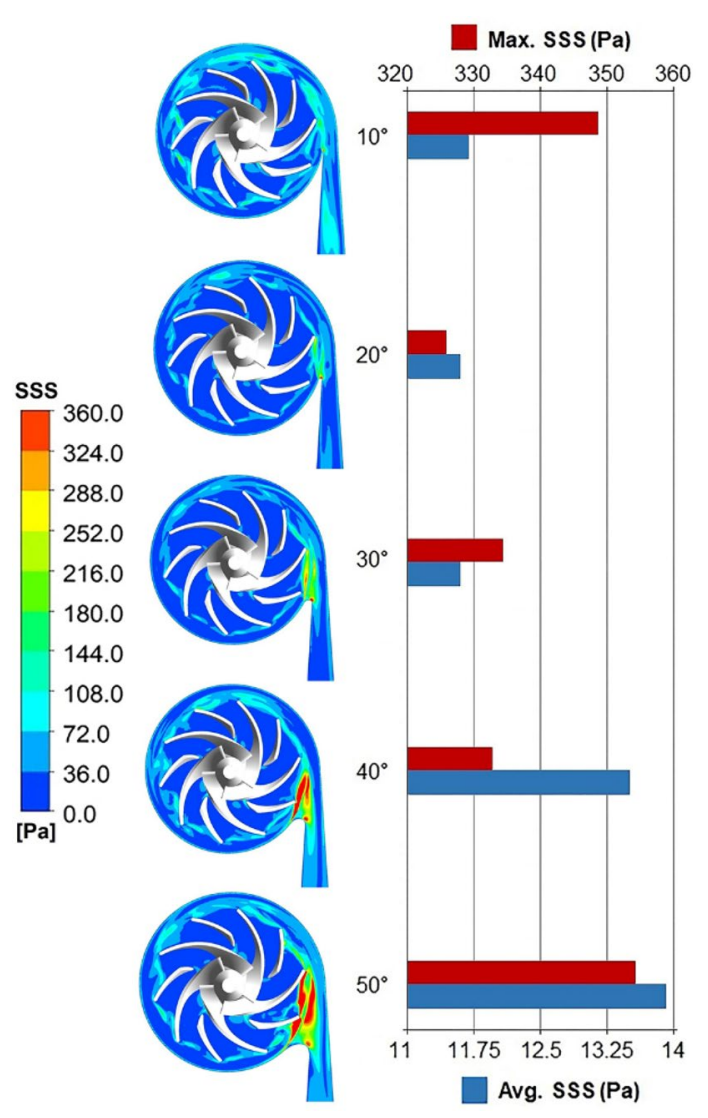

Fig. 6 The distribution of scalar shear stress at different tongue angles (left column), and the values of the maximum and averaged scalar shear stresses in the entire fluid domain. (right column)

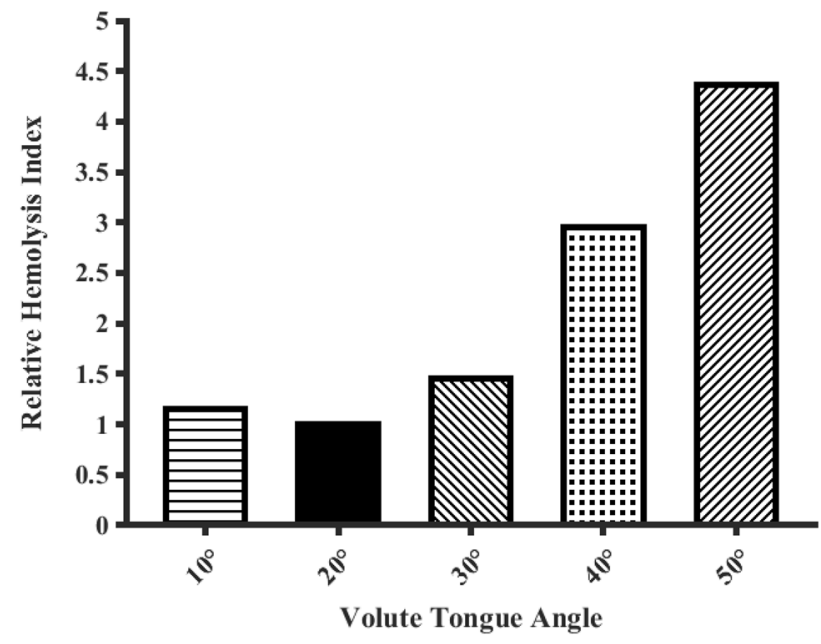

Fig. 7 Relative hemolysis index (normalized to the $20^{\circ}$ tongue angle)

contours indicated that increasing the tongue angle promotes the decrease in the corresponding net radial force on the impeller. However, the hydraulic efficiency and the head pressure decreased at higher tongue angles. Thus, the hydraulic experiments confirmed the in-silico characteristics of the pumps.

It was observed that the SSS distributions were negatively impacted by the larger tongue angle. Nevertheless, the lowest tongue angle $\left(10^{\circ}\right)$ generated a relatively high SSS value of $347.8 \mathrm{~Pa}$. This observation might be explained by the fact that too small of a tongue angle leads to an unusually sharp corner on the volute tongue region where the fluid is subjected to intense shear. Consequently, the intense shearing motion results in a high shear rate and excessive acceleration in a small area. The tongue angles of $10^{\circ}$ and $20^{\circ}$ were observed to be superior among the models regarding the hydraulic output and the blood damage.

Although the hydraulic output was slightly better for the $10^{\circ}$ tongue angle, $20^{\circ}$ tongue angle represents $13 \%$ lower hemolysis than that of $10^{\circ}$. However, the higher net radial force acting on the impeller was observed in the lower tongue angles. Near the volute tongue region and the trailing edge of blades, the blood damage risk is usually higher due to shear stress. Velocity vector fields showed that a large tongue angle resulted in flow separation. A well-matching volute tongue and impeller outflow should minimize the flow separation and the net radial force acting on the impeller. Eventually, a lower blood damage can be achieved. Henceforth, factors such as the hydraulic output, the net radial force and the amount of blood damage are critical indicators for pump designers.

The presented study reveals encouraging outputs, but there are also some limitations in the experimental study. the tolerances of pump geometry were around $100 \mu$ and removal of the support material was achieved by hand polishing. Maximum measurement errors of flow meter and pressure sensors were $\pm 0.15 \mathrm{~L} / \mathrm{min}$ and $\pm 1.875 \mathrm{mmHg}$, respectively, corresponds to the $0.5 \%$ of the full scale. Nevertheless, the hydraulic performance of the physical prototypes at the nominal condition was in good agreement with the in-silico analysis. Overall, the findings of this study do not necessarily put forth a definitive tongue angle in the pump design, but the reported effects of the tongue angle can serve as a guideline for the pump optimization. The tongue angle should be adjusted based on the design requirements such as hemolysis, hydraulic efficiency, magnetic suspension force, etc. Moreover, the reported radial force data is limited to highlighting the impact of volute tongue on the non-uniform pressure distribution around the impeller region. Further unsteady analyses at different flow rates might be needed to assess the magnetic suspension requirements for active levitation control. It should also be noted that the evaluation of the accuracy and credibility of the presented hemolysis estimation approach is beyond the scope of this study. This estimation 
method was adopted from the existing studies and implemented as a CFD tool for ranking the volute geometries.

\section{Conclusion}

In summary, this study explores impact of the volute tongue position on the hydraulic and hemolytic performance of a centrifugal blood pump. The tongue angle of $20^{\circ}$ demonstrated the minimum blood damage risk among five models $\left(10^{\circ}\right.$ to $\left.50^{\circ}\right)$ with an adequate hydraulic outputs. The blood damage potential and radial imbalance increases gradually as the tongue angle grows larger than $20^{\circ}$. Although the $10^{\circ}$ tongue angle represents the best hydraulic output and efficiency, yet it indicates slightly higher hemolysis index compared to $20^{\circ}$ tongue angle. Our findings showed a maximum increase in the hydraulic efficiency and pressure head up to $\% 9.1$ and $\% 8.8$ respectively when the volute tongue was modified. However, the same modifications resulted a substantial improvement of in-silico hemolysis estimation up to $77.8 \%$ at the design point.

This observation proves valuable for further research into this subject by repeating this study by considering the limitations. In our research we observed this by working on blood analogue fluid is used for the physical tests and the hydraulic efficiency was estimated from the in-silico findings. Also, depending on the impeller-volute interaction type and dimensional tolerance, the optimal volute tongue angle could vary significantly between different blood pump designs and further research is required considering this variation. Acknowledging these limitations, this study does not claim to establish a gold standard method for designing a volute geometry, rather it reveals the effect of the tongue position on a fixed impeller-varying volute tongue couple. Future studies with in-vitro blood tests and different impeller-volute examples may reveal further insights about the volute tongue position in LVAD design. Finally, this study provides an original insight to blood pump designers for finding the best volute tongue position to reduce the blood damage risk, increase the efficiency, minimize the power needs of a magnetic bearing or decrease the possible wear of the mechanical bearings.

Data availability and material All authors confirm that all data and materials as well as software application or custom code support their published claims and comply with field standards

\section{Compliance with ethical standards}

Conflicts of interest The authors declare that they have no conflict of interest.

SN Applied Sciences
Consent for publication All authors agreed with the content and that all gave explicit consent to submit and that they obtained consent from the responsible authorities at the institute/organization where the work has been carried out.

Open Access This article is licensed under a Creative Commons Attribution 4.0 International License, which permits use, sharing, adaptation, distribution and reproduction in any medium or format, as long as you give appropriate credit to the original author(s) and the source, provide a link to the Creative Commons licence, and indicate if changes were made. The images or other third party material in this article are included in the article's Creative Commons licence, unless indicated otherwise in a credit line to the material. If material is not included in the article's Creative Commons licence and your intended use is not permitted by statutory regulation or exceeds the permitted use, you will need to obtain permission directly from the copyright holder. To view a copy of this licence, visit http://creativecommons .org/licenses/by/4.0/.

\section{References}

1. Li H, Gou Z, Huang F, Ruan X-D, Qian W-W, Fu X (2019) Evaluation of the hemolysis and fluid dynamics of a ventricular assist device under the pulsatile flow condition [J]. J Hydrodyn 31(5):965-975

2. LiT-Y, Ye L, Hong F-W, Liu D-C, Fan H-M, Liu Z-M (2013) The simulation of multiphase flow field in implantable blood pump and analysis of hemolytic capability [J]. J Hydrodyn 25(4):606-615

3. Fan H-M, Hong F-W, Zhang G-P, Ye L, Liu Z-M (2010) Applications of CFD Technique in the Design and Flow Analysis of Implantable Axial Flow Blood Pump [J]. J Hydrodyn 22(4):518-525

4. Ozturk C, Aka IB, Lazoglu I (2018) Effect of blade curvature on the hemolytic and hydraulic characteristics of a centrifugal blood pump [J]. The Int J Artif Organs 41(11):730-737

5. Wong Y-W, Chan W-K, Hu W (2007) Effects of tongue position and base circle diameter on the performance of a centrifugal blood pump [J]. Artif Organs 31(8):639-645

6. Son CH, Jin HB, Kim MJ, Chung WJ (2014) Spiral casing of a volute centrifugal pump [J]. J Mech Sci Technol 28(7):2697-2706

7. Alemi H, Nourbakhsh SA, Raisee M, Najafi AF (2015) Effects of volute curvature on performance of a low specific-speed centrifugal pump at design and off-design conditions [J]. J Turbomach 137(4):041009-041010

8. Wu D, Yuan S, Ren Y, Mu J, Yang Y, Liu J (2016) CFD investigation of the influence of volute geometrical variations on hydrodynamic characteristics of circulator pump [J]. Chin J Mech Eng 29(2):315-324

9. Mozafari S, Rezaienia MA, Paul GM, Rothman MT, Wen P, Korakianitis T (2017) The effect of geometry on the efficiency and hemolysis of centrifugal implantable blood pumps [J]. ASAIO J 63(1):53-59

10. Taskin ME, Fraser KH, Zhang T, Wu C, Griffith BP, Wu ZJ (2012) Evaluation of eulerian and lagrangian models for hemolysis estimation [J]. ASAIO J 58(4):363-372

11. Peng Y, Wu Y, Tang X, Liu W, Chen D, Gao T, Xu Y, Zeng Y (2014) Numerical simulation and comparative analysis of flow field in axial blood pumps [J]. Comput Methods Biomech Biomed Eng 17(7):723-727

12. Malinauskas RA, Hariharan $P$, Day SW, Herbertson LH, Buesen $M$, Steinseifer U, Aycock KI, Good BC, Deutsch S, Manning KBJAJFDA (2017) benchmark medical device flow models for CFD validation [J]. ASAIO J 63(2):150-160 
13. Keshavarz-Motamed Z, Garcia J, Gaillard E, Capoulade R, Le Ven F, Cloutier G, Kadem L, Pibarot P (2014) Non-invasive determination of left ventricular workload in patients with aortic stenosis using magnetic resonance imaging and doppler echocardiography. PLoS ONE 9(1):e86793

14. Boehning F, Timms DL, Amaral F, Oliveira L, Graefe R, Hsu P-L, Schmitz-Rode T, Steinseifer U (2011) Evaluation of hydraulic radial forces on the impeller by the volute in a centrifugal rotary blood pump [J]. Artif Organs 35(8):818-825

15. Liu G-M, Jin D-H, Jiang X-H, Zhou J-Y, Zhang Y, Chen H-B, Hu S-S, Gui X-M (2016) Numerical and in vitro experimental investigation of the hemolytic performance at the off-design point of an axial ventricular assist pump [J]. ASAIO J 62(6):657-665

16. Stepanoff AJ (1958) Centrifugal and axial flow pumps theory design and application. Wiley Publishing, NY

17. Bludszuweit $C$ (1995) Model for a general mechanical blood damage prediction [J]. Artif Organs 19(7):583-589
18. Giersiepen M, Wurzinger LJ, Opitz R, Reul H (1990) Estimation of shear stress-related blood damage in heart valve prostheses-in vitro comparison of 25 aortic valves [J]. Int J Artif Organs 13(5):300-306

19. Heuser G, Opitz R (1980) A Couette viscometer for short time shearing of blood [J]. Biorheology 17(1-2):17-24

20. Farinas MI, Garon A, Lacasse D, N'Dri D (2006) Asymptotically consistent numerical approximation of hemolysis [J]. J Biomech Eng 128(5):688-696

21. Garon A, Farinas MI (2004) Fast three-dimensional numerical hemolysis approximation [J]. Artif Organs 28(11):1016-1025

Publisher's Note Springer Nature remains neutral with regard to jurisdictional claims in published maps and institutional affiliations. 